\title{
Research on Spectral Polarization Imaging Technique
}

\author{
Haibo Zhao ${ }^{1^{*}}$, Zheng Wang1, Xuling Lin'1, Huan Li ${ }^{1}$, Lei Feng ${ }^{2}$, Yu Zhou ${ }^{3}$ \\ ${ }^{1}$ Beijing Institute of Space Mechanics \& Electricity, Beijing, China \\ ${ }^{2}$ Beijing Institute of Spacecraft Environment Engineering, Beijing, China \\ ${ }^{3}$ DFH Satellite Co., Ltd., Beijing, China \\ Email: ${ }^{*}$ alabo1983@126.com
}

Received 16 October 2014

\begin{abstract}
The mechanism and characteristics of spectral polarization imaging technique are presented. The present research and developing trend of spectral polarization remote sensing are introduced. $A$ novel method of spectral polarization imaging technique is discussed, which is based on static intensity modulation adding with double refraction crystal spectrometer. The static intensity modulation consists of two retarders and one polarizer. The double refraction crystal is used to generate interference image. The spectral and four Stokes vectors information can be obtained only by one measurement. The method of static intensity modulation is deduced in detail and is simulated by computer. The spectropolarimeter experimental system is also established in the laboratory. The basic concept of the technique is verified.
\end{abstract}

\section{Keywords}

Spectral, Polarization, Stokes Vector, Intensity Modulation, Interference Image

\section{Introduction}

The intrinsic value of spectral polarization is remote sensing detection. Spectral polarization detection can obtain polarization information of high spatial resolution, high spectral resolution and polarization information. Spectral polarization detection can provide characteristic of objective. Spectral polarization remote sensing detection technology has significant applications value in the fields of atmospheric sounding, underwater detection, astronomical observation, resource investigation and military [1]. The summary of spectral polarization remote sensing method is presented, including the spectral polarization detection method, the mechanism and characteristics of polarization detection, and the expression of polarization light. It is an important developed direction based on the static intensity modulation adding with double refraction crystal spectrometer. The basic concept of the technique is verified based on simulation and experiment.

"Corresponding author. 


\section{Summary of Spectral Polarization Detection}

\subsection{Spectral Polarization Detection Method}

\section{(1) Linear polarization measurement using polarizer}

The measurement equipment is composed of optical system, polarizer, light filter and CCD. Using different filter can obtain different spectral image. Currently there are two measurement methods: using one camera and using three cameras. If using one camera, the image of linear polarization can be obtained by rotating the polarizer. One-camera system has the merits of simple configuration and small size, but it can't achieve real-time polarization image. Three-camera system has the merit of achieving real-time polarization image, but it has complex configuration and big size.

(2) Polarization modulation method

Polarization modulation method can be classified as two types: polarization device rotating and phase modulation. Polarization device rotating: the phase of incident light is modulated by rotating the $1 / 4$ waveplate and the polarizer. Stokes vector can be solved by the intensity of different modulation state. Phase modulation: Electro-Optical phase modulator is used instead of the $1 / 4$ waveplate. Phase is modulated by varying the voltage applied across the phase modulator. There is no mechanism rotating during measuring but the real-time polarization image can't be achieved. The electric noise and the modulation error seriously influence the detection precision. Precise calibration is needed before measuring. With the use of LCTF/AOTF can obtain spectral information.

(3) Static intensity modulation

Basic concept of the technique [2]: the incident light is modulated by two retarders and one polarizer, and then the light signal is obtained by the spectrometer. The retardation of the two retarders is different and the proportion is about 1:2 or other numbers. The Stokes vector of each spectral band can be solved based on frequency domain algorithm. There is no mechanism rotating during measuring.

\subsection{Mechanism and Characteristics of Polarization Detection}

The direction and magnitude of electric vector of polarized light change regularly. Any polarized light can be expressed by two linear polarized light of orthogonally oscillating and correlative phase [3]. The sunlight is isotropic. The vertical and horizontal components of the reflective light change after the sunlight irradiates the earth object. Then the light will become partial polarized light or polarized light [4]. The polarization state of the reflective light could be influenced by surface configuration of object, texture and incident angle of light. Furthermore, the thermal radiance of object also has polarization effect. The characteristic of object can be obtained by the change of light polarization and thereby distinguishing object could be more effective.

Compared with traditional optics and radiation remote method, polarization detection method has three merits [5]: polarization detection can solve some problem which can't be solved by traditional method, such as the problem of the particle distributing of cloud and aerosol; polarization detection can achieve high resolution without detecting exact radiation; the radiation measurement data and the polarization information can be obtained simultaneously.

\subsection{Expression of Polarization Light}

There are two methods to express polarization state of light: Jones vector and Stokes vector. Jones vector is used to express complete polarization light; Stokes vector can express any polarization state and its parameters are all real numbers. It is convenient to measure the parameters of Stokes vector. Stokes vector is used frequently in the field of polarization remote sensing. The expression of Stokes vector is (normalized) [6]:

$$
S= \begin{cases}I=I_{0}+I_{90}=I_{45}+I_{135}=I_{R}+I_{L} & (0 \leq I \leq 1) \\ Q=I_{0}-I_{90} & (-1 \leq Q \leq 1) \\ U=I_{45}-I_{135} & (-1 \leq U \leq 1) \\ V=I_{R}-I_{L} & (-1 \leq V \leq 1)\end{cases}
$$

where:

$I=$ total light intensity

$Q=$ intensity difference between horizontal and vertical linearly polarized components 
$U=$ intensity difference between linearly polarized components oriented at \pm 45

$V=$ intensity difference between right and left circular components

$$
\begin{cases}P=\frac{\sqrt{Q^{2}+U^{2}+V^{2}}}{I} & (0 \leq P \leq 1) \\ \varphi=\frac{1}{2} \tan ^{-1}\left(\frac{U}{Q}\right) & \left(-90^{\circ} \leq \varphi \leq 90^{\circ}\right)\end{cases}
$$

where:

$P=$ degree of polarization (DOP)

$\varphi=$ azimuth of the major axis of a polarization ellipse

$P$ is a real number between 0 and $1 . P=0$ indicates non-polarized light; $P=1$ indicates complete polarized light. $0<P<1$ indicates partial polarized light. Even if the light intensity changes, $P$ and $\varphi$ don't change:

$$
\left\{\begin{array}{l}
P^{\prime}=\frac{\sqrt{Q^{\prime 2}+U^{\prime 2}}}{I^{\prime}}=\frac{\sqrt{(k Q)^{2}+(k U)^{2}}}{k I}=\frac{\sqrt{Q^{2}+U^{2}}}{I}=P \\
\varphi^{\prime}=\frac{1}{2} \tan ^{-1}\left(\frac{U^{\prime}}{Q^{\prime}}\right)=\frac{1}{2} \tan ^{-1}\left(\frac{k U}{k Q}\right)=\frac{1}{2} \tan ^{-1}\left(\frac{U}{Q}\right)=\varphi
\end{array}\right.
$$

\section{Development of Spectral Polarization Remote Sensing Technology}

The domestic research of spectral polarization remote sensing technology started late. Many problems of theory and experiment are needed to be researched. The typical product is multi-band polarization CCD camera designed by Anhui Institute of Optics and Fine Mechanics, Chinese Academy of Sciences. The camera has three parallel optics systems which synchronously collect polarization images of 0,60, 120 degree. Linear polarization information can be obtained. Four spectral bands are 443, 555, 665, $865 \mathrm{~nm}$ and the bandwidth is 30-50nm. Characteristic of the instrument: the focus is $200 \mathrm{~mm}$, the detector is $1024 \times 1024$ area array CCD, data quantization is 12 bits [7].

The research of spectral polarization remote sensing technology is mature in many other countries. Some research institutions and companies have designed many polarization detection instruments. For example, there are US Army Research Laboratory (ARL), Centre National d'Etudes Spatiales (CNES, France) and Ball Aerospace \& Technologies Corp.

A compact, lightweight, robust, and field-portable spectropolarimetric imaging system was developed at ARL. It operates in the 400 to $900 \mathrm{~nm}$ region with a bandwidth of $10 \mathrm{~nm}$ at $600 \mathrm{~nm}$. This automated imager is designed using a tellurium dioxide $\left(\mathrm{TeO}_{2}\right)$ acousto-optic tunable filter (AOTF) as an agile spectral selection element and a commercial nematic liquid crystal variable retardation (LCVR) plate as a tunable polarization selection device with an un-cooled charge coupled device (CCD) camera. Image acquisition with both spectral and polarization features facilitates significant improvement in target detection [8].

POLDER of CNES is composed of polarizer and light filter. It can detect global radiation and polarization information of aerosol and cloud. There are nine spectral bands (443 - $1020 \mathrm{~nm})$. Three bands (490, 670 and 865 $\mathrm{nm})$ are polarization channels, six bands are radiation channels [9].

The BATC Stokes imaging polarimeter was designed by Ball Aerospace \& Technologies Corp. The polarimeter employs electro-optic modulators to acquire full Stokes vector spectral image (LCTF: liquid crystal tunable filter, LCVR: liquid crystal variable retarders) [10].

\section{Novel Method of Spectral Polarization Detection}

The novel method of spectral polarization imaging technique is based on static intensity modulation adding with double refraction crystal spectrometer. The static intensity modulation consists two retarders and one polarizer. The double refraction crystal is used to generate interference image (Figure 1).

The interference image is detected by area CCD and the digital signal can be obtained. The digital signal is auto correlative function $C(h) . C(h)$ is made up of $A_{0}(h), A_{0}\left(h-\left(L_{2}-L_{1}\right)\right)$, and $A_{2}\left(h-L_{2}\right) . L_{1}$ and 
$L_{2}$ is the optical path difference (OPD) of retarder 1 and retarder 2. $\Phi_{1}(\sigma)$ and $\Phi_{2}(\sigma)$ is the retardation of retarder 1 and retarder 2 ( $\sigma$ is wave number). $F\{\}$ means Fourier transform. $S_{0}(\sigma), S_{1}(\sigma), S_{2}(\sigma)$ and $S_{3}(\sigma)$ can be calculated by:

$$
\begin{gathered}
F\left\{A_{0}(h)\right\}=\left[\frac{1}{2}\right] \times S_{0}(\sigma) \\
F\left\{A_{1}\left[\left(h-\left(L_{2}-L_{1}\right)\right)\right]\right\}=\left[\frac{1}{8} \mathrm{e}^{i \times\left[\phi_{2}(\sigma)-\phi_{1}(\sigma)\right]}\right] \times\left\{S_{2}(\sigma)+i \times S_{3}(\sigma)\right\} \\
F\left\{A_{2}\left(h-L_{2}\right)\right\}=\left[\frac{1}{4} \mathrm{e}^{i \times \phi_{2}(\sigma)}\right] \times S_{1}(\sigma)
\end{gathered}
$$

The method of static intensity modulation is simulated by computer. $S_{0}(\sigma), S_{1}(\sigma), S_{2}(\sigma)$ and $S_{3}(\sigma)$ is simulated as shown in Figure 2. The signal $C(h)$ is simulated as shown in Figure 3. $S_{0}(\sigma), S_{1}(\sigma)$, $S_{2}(\sigma)$ and $S_{3}(\sigma)$ can be calculated by function (4)-(6). The simulation RMS error of DOP is about $1 \%$ as shown in Figure 4.

The experimental system is also established in the laboratory: the static intensity modulation adding with the spectrometer. The basic concept of the technique is verified.

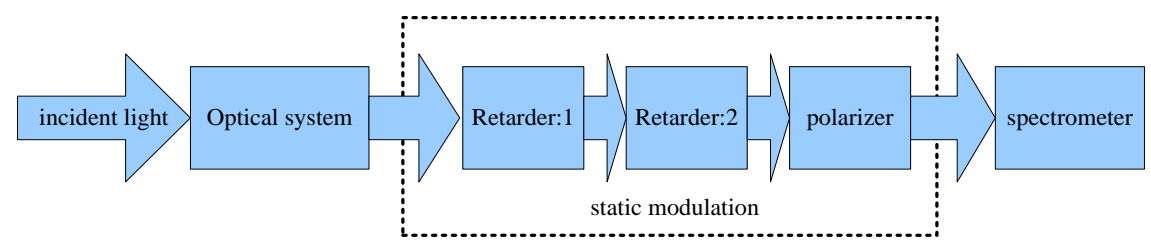

Figure 1. Basic concept of static intensity modulation.

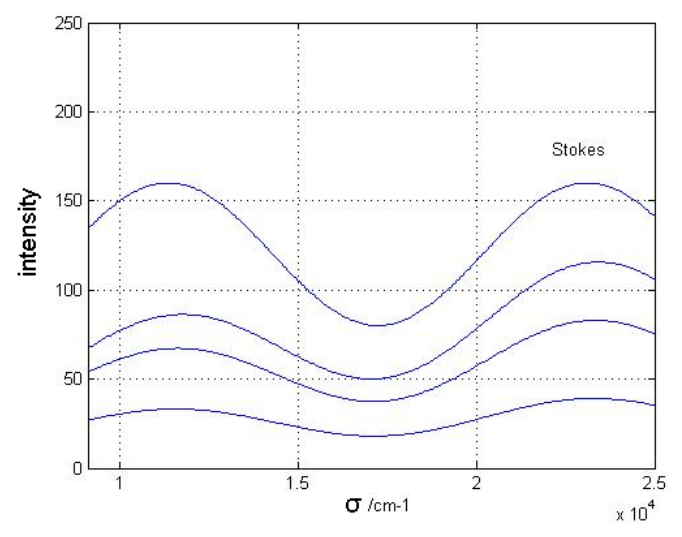

Figure 2. Simulation of $S_{0}(\sigma), S_{1}(\sigma), S_{2}(\sigma)$ and $S_{3}(\sigma)$.

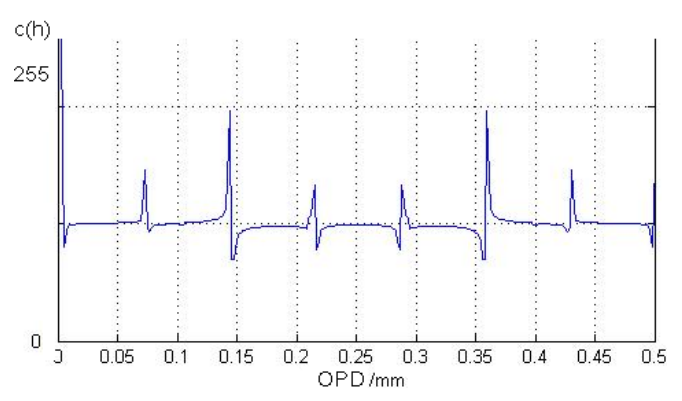

Figure 3. Simulation of signal $C(h)$. 


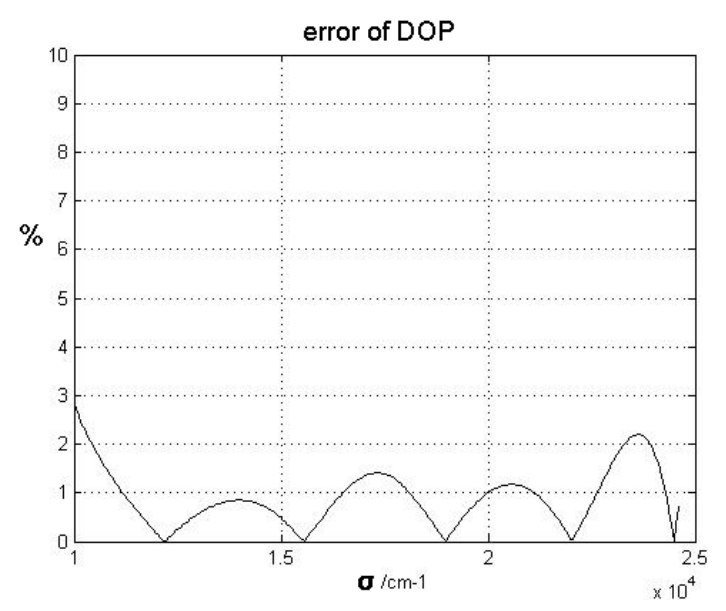

Figure 4. Simulation error of DOP.

\section{Conclusion}

A novel method of spectral polarization imaging technique is discussed, which is based on static intensity modulation adding with double refraction crystal spectrometer. The imaging system can obtain spectral and polarization information. The advantages of the imaging system are compactness, low mass, no moving parts, and no electrical control. The system structure and basic theory are introduced. The experimental system is established in the laboratory. The basic concept of the system is verified. The simulation RMS error of polarization degree detection is about $1 \%$. The image data captured by the system can be applied to object identification and object classification.

\section{Funding}

Project is supported by the National Natural Science Foundation of China (Grant No. 11204014).

\section{References}

[1] Yang, Z.W. (2003) Measurement and Achievement of Polarimetric Spectrum. Chinese Journal of Spectroscopy Laboratory, 20, 815-820.

[2] Li, J., Zhu, J.P., Qi, C., et al. (2013) Static Fourier-Transform Spectral Imaging Full Polarimetry. Acta Phys. Sin, 62, 044206.

[3] Yu, D.Y. and Tan, H.Y. (1999) Engineering Optics. China Machine Press, Beijing, 322.

[4] Tan, Z.J., Li, J. and Cheng, H.Q. (2007) Application of Polarization Measurement in the Identification of the Space Target. Laser \& Infrared, 37, 200-203.

[5] Zhang, C.Y., Cheng, H.F., Chen, Z.H., et al. (2007) The Present Research and Developing Trend of Polarization Remote Sensing. Laser \& Infrared, 37, 1237-1240.

[6] Tang, R.Y., Yu, G.P. and Wang, X.F. (2006) Acquisition Method of Polarization Image in Natural Lighting Environment. J. Wuhan Univ., 52, 59-63.

[7] Sun, X.B., Hong, J. and Qiao, Y.L. (2005) The Study of Method for Feature Extraction Based on Angle of Polarization Parameter Image. Remote Sensing Technology and Application, 20, 256-260.

[8] US Army Research Laboratory (2003) Acousto-Optic Tunable Filter Based Visible- to Near-Infrared Spectropolarimetric Imager. Opt. Eng., 41, 1033-1038.

[9] Deschamps, P.-Y., Brdon, F.C.-M., Leroy, M., et al. (1994) The POLDER Mission: Instrument Characteristics and Scientific Objectives. IEEE, 32, 598-615.

[10] Ball Aerospace \& Technologies Corp. (2005) High-Speed Fieldable Imaging Stokes Vector Polarimeter. SPIE, 5888, 0x1-0x10. 